\title{
The effects of trauma on children: working to define roles for mental health professionals
}

\author{
Daniel S. Pine
}

National Institute of Mental Health, 15K North Drive, Room 110, MSC 2670, Bethesda, MD 20892-1670, USA, email Daniel.pine@nih.gov

nterest concerning the effects of trauma on the mental health of children has grown markedly during the past few years. At least three factors contribute to this growing concern.

First, studies in the basic sciences suggest that interactions between experiential and genetic factors during particular stages of development can exert long-term influences on a set of behaviours and physiological responses related to stress regulation (M eaney, 2001; $\mathrm{G}$ ross et al, 2002). Results from studies of people with various psychiatric conditions have begun to raise important questions concerning the degree to which similar developmental relationships arise in our species (C aspi et al, 2002; Heim \& N emeroff, 2002).

Second, advances in assessment and epidemiological methods have facilitated a series of prospective investigations of the sequelae of childhood trauma (Pine $\&$ Cohen, 2002). Studies in this area generate concerns over the high rates of trauma exposure and the strong associations with psychopathology.

Finally, particular interest in trauma follows in the wake of the recent terrorist attacks in the U nited States. As with data from other recent exposures of large groups of children to trauma, these events raise basic questions about the degree to which children may be particularly vulnerable to psychological harm following trauma (Laor et al, 1997; Schlenger et al, 2002; Thabet et al, 2002).

Given such concerns, recent reviews have considered in detail the data from children on levels of exposure to trauma, moderators of risk, effects on physiology and available treatments (Kaufman et al, 2000; Breslau, 2002; Pine $\&$ Cohen, 2002). The present article briefly summarises the conclusions from these prior reports, while emphasising three aspects of the available literature:

o the prevalence of trauma exposure, the nature of asso ciated risks for psychopathology, and the factors most consistently shown to moderate outcomes

o the results of randomised controlled treatment trials that inform therapeutics in traumatised children

o strategies for minimising risk for psychopathology following trauma.

\section{The nature of exposure}

$H$ eterogeneity in the nature of traumatic exposure, the contexts of exposures and the nature of psychopathology complicate efforts to draw firm conclusions on the risks of psychological sequelae in children. Reported prevalence rates of childhood trauma exposure generally exceed
$20 \%$ in most studies of representative samples from industrial countries, and frequently exceed $50 \%$ in studies from countries or contexts associated with various forms of political unrest or social instability (which increase risks for trauma).

Children experience trauma through exposure to a diverse array of adverse events, including episodes of domestic violence, natural disasters and political unrest, each of which has been shown markedly to increase the risk of psychological problems (Breslau, 2002; Pine \& Cohen, 2002). Key aspects of traumatic experiences vary widely across this array of events. It remains unclear whether conclusions can be drawn concerning a common set of risks and moderators of outcome that apply across diverse forms of trauma, such as domestic violence as well as political violence.

Some types of trauma have a particularly heightened risk for psychopatho lo gy. In general, across the full range of traumatic exposures, two factors most consistently moderate risk in prospective investigations: overall level of exposure and the degree of social support. D ata on social support appear particularly informative, in that they suggest that children experience the greatest need for intervention when trauma - such as natural disasters, domestic violence or political unrest - occurs in the context of disrupted social support networks. Findings have emerged for other potential moderators, such as age at the time of exposure or level of pre-exposure psychopathology, but these remain only suggestive, as clear associations have not emerged in all studies.

Finally, studies of diverse traumatic events consistently find strong associations between trauma and psychopathology, but disagreement persists on the degree to which risk is elevated for one or another specific condition (Steinberg \& Avenevoli 2000; Breslau, 2002). Some preliminary evidence suggests that risk for vario us conditions may meaningfully vary across age groups and contexts. For example, adolescents may be more likely than young children to manifest the constellation of symptoms that characterises post-traumatic stress disorder in adults. N evertheless, strong evidence has not emerged of selectively elevated risk for specific conditions asso ciated with specific forms of trauma or particular developmental periods; strong associations appear with a wide range of conditions, including various mood and anxiety disorders, as well as various behaviour disorders (Steinberg \& Avenevoli, 2000; Breslau, 2002; Pine $\&$ C ohen, 2002).

Given the complicated nature of associations between trauma and potentially comorbid mental health problems,
Studies of diverse traumatic events consistently find strong associations between

trauma and psychopathology, but disagreement persists on the degree to which risk is elevated for

one or another specific condition.

\section{Some types of \\ trauma have a particularly heightened risk for psycho- pathology. In general, across the \\ full range of traumatic expo- sures, two factors most consistently moderate risk in \\ prospective investigations: overall level of exposure and the degree of social support.}

The opinions and assertions contained in this paper are the private views of the author and are not to be construed as official or as reflecting the views of the $\mathrm{N}$ ational Institute of Mental Health or the US Department of Health and Human Services. 
children exhibiting clearly impairing psychological problems following exposure to trauma should be assessed by mental health practitioners experienced in evaluating children with symptoms in multiple domains.

\section{Therapeutics}

Since traumatic experiences predispose to a diverse array of conditions, recent broad advances in treatment approaches for many paediatric mental disorders may address some essential questions on therapeutics. Specifically, recent large-scale randomised controlled trials (RCTs) for paediatric mood, anxiety and behaviour disorders have documented marked benefits for both psychopharmacological and psychotherapeutic interventions (Emslie \& Mayes, 2001; Jensen et al, 2001; Research Unit on Pediatric Psychopharmacology Anxiety Study Group, 2001). In general, conclusions from these studies on the relative merits of vario us treatments for specific psychological problems can be applied to children exhibiting behaviour, mood or anxiety disorders within the context of trauma. Again, given the potential complexity of treatment decisions for children with comorbid problems following traumatic exposures, mental health practitioners play an invaluable role in using data from available studies to weigh the risks and benefits of particular treatments for particular children.

The psychological problems of traumatised children do clearly pose unique questions concerning therapeutics, necessitating studies that specifically examine the relative merits of interventions. Unfortunately, RCTs have examined the efficacy of only a narrow range of treatments applied in a narrow range of circumstances. Specifically, at least six studies document the efficacy of cognitivebehavioural therapy (CBT) for symptoms arising following sexual abuse, and one small study documents the benefits of imipramine for acute stress disorder following a severe burn (see Pine \& Cohen, 2002). Major questions in other areas remain unanswered. For example, no RCT has examined the efficacy of selective serotonin reuptake inhibitors, which have been shown to benefit adults with post-traumatic stress disorder and children with a range of mood or anxiety disorders. In fact, no RCT has examined the efficacy of any treatment directed at specific mental disorders that arise following any form of trauma beyond sexual abuse. Given concerns with tricyclic antidepressant use among children and limitations in other areas of therapeutic research, the only current, generally accepted treatment recommendation specifically for traumatised children involves targeting anxiety symptoms with CBT in initial interventions (Pine $\&$ Cohen, 2002). Decisions on other interventions should be based on the larger literature on therapeutics of paediatric mental syndromes.

\section{Minimising risk}

Insufficient data exist to inform preventive interventions, either for specific children exposed to episodes of domestic abuse or for large groups of children exposed to massive acute traumas, such as the attacks on 11
September 2001. Ideally, firm recommendations will be based on data from randomised controlled prevention trials in similarly traumatised populations. Rising concerns both with the effects of domestic abuse and with the risk for future acts of terrorism may increase efforts to implement such prevention trials.

In the absence of such data, caution seems warranted, for a few reasons. Studies in traumatised adults suggest that potential preventive interventions can exert unanticipated deleterious effects (Rauch et al, 2001). Similarly, prevention studies among non-traumatised children also document the potential for deleterious effects from mass interventions. Specifically, lessons from past efforts to address rising adolescent suicide rates may be relevant. In this area, initial results suggested that strategies involving the identification and treatment of those children most in need may ultimately prove more beneficial than wide-scale, non-selective, population-based interventions (Shaffer \& Craft, 1999). School-based or primary care settings may employ people with limited mental health expertise to identify those children most in need of assessments by mental health professionals. $D$ ata on moderators of risk from epidemiological studies of trauma exposure can be used to inform such case-finding strategies. Specifically, parents, educators and primary care physicians should be most concerned with children who either receive high levels of exposure to trauma or who are exposed to trauma within the context of marked disruption in social support. Efforts to prevent sequelae of trauma in children might consider the merits of working to identify such children and use insights from recent research on therapeutics of various paediatric mental syndromes to provide relief of emergent symptoms. Similarly, prevention efforts might consider the merits of interventions by parents, educators and primary care providers that either limit secondary exposure, for example through media exposures, or address social disruption following trauma.

\section{Conclusions}

In closing, concern with the psychiatric sequelae of trauma follows from diverse findings in both the scientific and lay press. Accumulating knowledge from epidemiological studies generates specific concerns over the degree to which recent widely publicised traumatic events might prove particularly harmful to the psychological well-being of children. Available data document the nature of such risks and factors that moderate outcomes. While advances in therapeutics provide insights for general treatment approaches, considerable research is needed on both therapeutics for and prevention of disorders that specifically arise following traumatic experiences.

\section{References}

Breslau, N . (2002) Psychiatric morbidity in adult survivors of childhood trauma. Seminars in Clinical Neuropsychiatry, 7, 8088.

Caspi, A., McClay, J., Moffitt, T. E., et al (2002) Role of genotype in the cycle of violence in maltreated children. Science, 297, $851-854$ 
Emslie, G. J. \& Mayes, T. L. (2001) Mood disorders in children and adolescents: psychopharmacological treatment. Biological Psychiatry, 49, 1082-1090.

Gross, C., Zhuang, X., Stark, K., et al (2002) Serotonin SA $_{1 \mathrm{~A}}$ receptor acts during development to establish normal anxietylike behaviour in the adult. Nature, 416, 396-400.

Heim, C. \& N emeroff, C. B. (2002) N eurobiology of early life stress: clinical studies. Seminars in Clinical Neuropsychiatry, 7, 147-159.

Jensen, P. S., H inshaw, S. P., Swanson, J. M., et al (2001) Findings from the $\mathrm{NIMH}$ Multimodal Treatment Study of ADHD (MTA): implications and applications for primary care providers. Journal of Developmental and Behavioral Pediatrics, 22, 60-73.

Kaufman, J., Plotsky, P. M., N emeroff, C. B., et al (2000) Effects of early adverse experiences on brain structure and function: clinical implications. Biological Psychiatry, 48, 778-790.

Laor, N., Wolmer, L., Mayes, L. C., et al (1997) Israeli preschool children under Scuds: a 30-month follow-up. Journal of the American Academy of Child and Adolescent Psychiatry, 36, 349-356.

Meaney, M. J. (2001) Maternal care, gene expression, and the transmission of individual differences in stress reactivity across generations. Annual Review of Neuroscience, 24, 1161-1192.
Pine, D. S. \& Cohen, J. A. (2002) Trauma in children and adolescents: risk and treatment of psychiatric sequelae. Biological Psychiatry, 51, 519-531.

Rauch, S. A., Hembree, E. A. \& Foa, E. B. (2001) Acute psychosocial preventive interventions for posttraumatic stress disorder. Advances in M ind-Body Medicine, 17, 187-191.

Research U nit on Pediatric Psychopharmacology Anxiety Study Group (2001) Fluvoxamine for the treatment of anxiety disorders in children and adolescents. New England Journal of M edicine, 344, 1279-1285.

Schlenger, W. E., Caddell, J. M., Ebert, L, et al (2002) Psychological reactions to terrorist attacks: findings from the $\mathrm{N}$ ational Study of Americans' Reactions to September 11. Journal of the American Medical Association, 288, 581-588.

Shaffer, D. \& C raft, L. (1999) Methods of adolescent suicide prevention. Journal of Clinical Psychiatry, 60 (suppl. 2), 70-74; discussion 75-76, 113-116.

Steinberg, L. \& Avenevoli, S. (2000) The role of context in the development of psychopathology: a conceptual framework and some speculative propositions. Child Development, 71, 66- 74.

Thabet, A. A., Abed, Y. \& Vostanis, P. (2002) Emotional problems in Palestinian children living in a war zone: a cross-sectional study. Lancet, 359, 1801-1804.

\section{Impact of trauma on Palestinian children's mental health: lessons from the Gaza studies}

\section{Panos Vostanis}

Professor of Child and Adolescent Psychiatry, University of Leicester, Westcotes House, Westcotes Drive, Leicester LE3 0QU, UK, email pv11@le.ac.uk

hildren exposed to violence are at high risk of developing a range of mental health problems, predominantly post-traumatic stress disorder (PTSD) and depression (Yule, 1999). Children in war zones can be affected not only directly but also indirectly, for example through their basic health needs not being met, the loss of family members, disruption of social networks, internal displacement and their parents' responses.

Since the late 1990s, we have run a number of studies in the Gaza Strip, with Dr Abdel Aziz Thabet and his research team. 0 ur initial study, after the end of the first intifada (uprising), established a high prevalence rate (41\%) of post-traumatic stress reactions among children (aged 611 years) and their significant association with traumatic events experienced by the children, as well as with other behavioural and emotional problems (Thabet \& Vostanis, 1999a). The trauma experienced included injuries, death or imprisonment of family, relatives or friends, as well as day and night raids.

When we re-interviewed these children $(n=234)$ 1 year later, well atter the 0 slo agreement and the renewal of the peace process in the region, the prevalence rate of post-traumatic stress reactions had decreased to $10 \%$ (Thabet \& Vostanis, 2000). The findings suggested that most reactions were acute and resolved in the absence of further conflict, but there were also a substantial number of children who suffered chronic and resistant posttraumatic reactions, which could benefit from specialist treatment.

Palestinian children's mental health problems were compounded by their extreme adverse socio-economic circumstances, which is a common finding in research with children who are victims of political conflict. Most traumatic events occur in refugee camps in the West Bank and the Gaza Strip, where generations of children have been born for 55 years with little hope of escape. There has been a continuous growth of the population, which has now reached a density of $2300 / \mathrm{km}^{2}$. Refugees make up $63 \%$ of the population, and $51 \%$ of refugees are under 15 years of age (Thabet \& Vostanis, 1999b).

The second intifada (Al Aqsa) started in September 2000. Although we could not access all areas of the $G$ aza Strip to follow up the previous sample, we repeated the epidemiological study with a new cohort, and found that the prevalence rates of post-traumatic stress reactions had again risen dramatically, as a consequence of high exposure to new traumatic events. Children's emotional presentations were strongly correlated with maternal psychopathology (i.e. mothers' response to trauma) (Thabet et al, 2001). Traumatic events had changed since the previous confilict, with most children reporting watching pictures of mutilated bodies on television, and witnessing the bombing of people and houses.
Palestinian children's mental health problems were compounded

by their extreme adverse socioeconomic circumstances, which is a common finding in research with children who are victims of political conflict. 